No factors other than those shown above were found in any of the cases tried, except for the algebraic factors and the factors less than 300,000 which appear in Cunningham and Woodall [1]. Check runs were not made, but it appears to be quite unlikely that any factor less than $2^{30}$ was missed.

Appendix. At the suggestion of the referee, two lists are included which show the progress which has been made in factoring numbers of the form $2^{n} \pm 1$. These lists have been prepared with the help of D. H. Lehmer and J. L. Selfridge. First we have a list of all the cases we could find in which complete factorizations have been claimed, where for $2^{n}-1$ only odd values of $n$ are considered.

$$
\begin{aligned}
& 2^{n}-1: n=1-99,105,107,111,113,115,117,123,127,129,135,151,521,607 \text {, } \\
& 1279,2203,2281 \\
& 2^{n}+1: n=0-102,105,106,108,110,111,112,113,114,118,120,122,123,126 \text {, } \\
& 130,134,135,138,146^{*}, 148,150,154,162,170,174,182,186^{*}, 190^{*} \text {, } \\
& 198,210,234^{*}, 270
\end{aligned}
$$

Some of these were not known to the author at the time the work described above was carried out. It should be mentioned that in several cases there is doubt that the factorizations are in fact complete; this is true, in particular, in the cases marked with an asterisk. Notice that M. Kraitchik [6] had already given a supposedly complete factorization of $2^{95}-1$, but that we found above that a further decomposition of one of his factors is possible.

The second list concerns the "original Mersenne numbers" (that is, numbers of the form $2^{p}-1$ where $p$ is prime and $p \leq 257$ ), and brings up to date a similar list by R. C. Archibald in $M T A C$ [7].

$$
p
$$

$2,3,5,7,13,17,19,31,61,89,107,127$

(All other $p<100), 113,151$

$163,173,179,181,223,233,239,251$

$109,131,157,167,191,197,211,229$

$101,103,137,139,149,193,199,227,241,257$

University of California

Berkeley, California

\begin{abstract}
Character of $2^{p}-1$
Prime

Composite and completely factored Two or more prime factors known

Only one prime factor known

Composite but no factor known
\end{abstract}

Raphael M. Robinson

The operation of this computer is supported in part by the National Science Foundation.

1. A. J. C. Cunningham \& H. J. Woodall, Factorisation of $y^{n} \mp 1, y=2,3,5,6,7,10$, 11, $12 u p$ to high powers $(n)$, Francis Hodgson, London, 1925.

2. D. H. Lehmer, "On the factors of $2^{n} \pm 1$," Amer. Math. Soc., Bull., v. 53, 1947, p. 164-167.

3. Raphael M. Robinson, "Mersenne and Fermat numbers," Amer. Math. Soc., Proc., v. 5 , 1954 , p. 842-846.

4. Raphael M. Robinson, "Factors of Fermat numbers," $M T A C$, v. 11, 1957, p. 21-22.

5. J. L. Selfridge, "Factors of Fermat numbers," $M T A C$, v. 7, 1953, p. 274-275.

6. M. KRAITCHIK, Introduction à la Théorie des Nombres, Gauthier-Villars, Paris, 1952. p. 39.

7. R. C. ARChibald, "Mersenne numbers," $M T A C$, v. 3, Note 98, 1949, p. 398.

\title{
On the Solution of "Jury" Problems with Many Degrees of Freedom
}

1. Introduction. In a recent numerical investigation using the Differential Analyser, it was found necessary to solve differential equations of up to the eighth order with two-point boundary conditions, the so-called "Jury" problem. Now, 
for second order equations with only one degree of freedom various processes of inverse interpolation may be used to estimate, from trial solutions, the correct initial conditions for the required solution. Of these, perhaps the best for this purpose is that due to Aitken $[1,2]$, which is, as it stands, equally suitable for direct or inverse interpolation. An example of its application is given in section 2 .

The method given below is an extension to many variables of a similar process. If we have $n$ functions $f(u, v, \cdots, z), g(u, v, \cdots, z), \cdots, m(u, v, \cdots, z)$, of the same $n$ independent variables, it is necessary to interpolate between $n+1$ sets of values of $f, g, \cdots, m$, to find the $n$ values of $u, v, \cdots, z$, required to form the wanted values of the functions. With more than one variable we are restricted to linear interpolates only, as the extension to higher order interpolates turns out to be unprofitable and difficult to generalise. It is found to be simpler to use the process of linear inverse interpolation iteratively, obtaining from the first $n+1$ sets of values a further set based on the first linear interpolation, then using this and the best $n$ preceding sets to repeat the process.

2. Aitken's process for one variable. This may be illustrated by means of an example of a "Jury" problem. Consider the differential equation

$$
\frac{d^{2} x}{d t^{2}}+\frac{d x}{d t}+x^{2}=0
$$

where the solution is required satisfying the boundary conditions

$$
x(u)=a, x(v)=b .
$$

We may then integrate forward from $t=u$ with two arbitrary initial values of $x^{\prime}$, say $x_{1}{ }^{\prime}$ and $x_{2}{ }^{\prime}$, to reach $t=v$, when $x=c_{1}$ and $c_{2}$ respectively. Defining $d=c-b$, we may interpolate $d=0$ to find, in the usual notation,

$$
x_{3}{ }^{\prime}=x^{\prime}\left(0 ; d_{1}, d_{2}\right) \text {. }
$$

as a starting value of $x^{\prime}$ for a new run. This produces $d_{3}$, and further interpolation yields both $x^{\prime}\left(0 ; d_{1}, d_{3}\right)$ and $x^{\prime}\left(0 ; d_{1}, d_{2}, d_{3}\right)$. The sequence of operations may be shown as:

$$
\begin{aligned}
& \begin{array}{lll}
x_{1}{ }^{\prime} & c_{1} & d_{1}
\end{array} \\
& x_{2}^{\prime} \quad c_{2} \quad d_{2} \quad x^{\prime}\left(0 ; d_{1}, d_{2}\right)=x_{3}{ }^{\prime} \\
& x_{3}{ }^{\prime} \quad c_{3} \quad d_{3} \quad x^{\prime}\left(0 ; d_{1}, d_{3}\right) \quad x^{\prime}\left(0 ; d_{1}, d_{2}, d_{3}\right)=x_{4}{ }^{\prime} \\
& x_{4}{ }^{\prime} \quad c_{4} \quad d_{4} \quad x^{\prime}\left(0 ; d_{1}, d_{4}\right) \quad x^{\prime}\left(0 ; d_{1}, d_{2}, d_{4}\right) \quad x^{\prime}\left(0 ; d_{1}, d_{2}, d_{3}, d_{4}\right)=x_{5}{ }^{\prime} \\
& x_{5}{ }^{\prime}
\end{aligned}
$$

and so on.

It should be noted in passing that convergence is not guaranteed, since a solution of the type we want may not exist. Results for the above equation with the boundary conditions $x(0)=1, x(1)=0$ are shown in Table 1 .

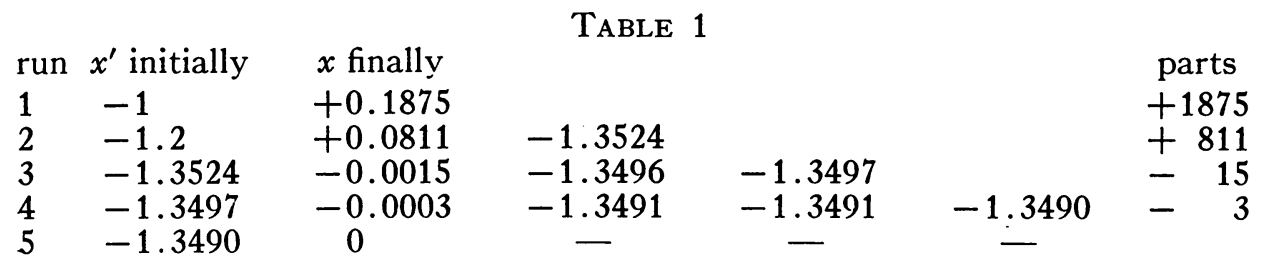


3. Extension to many variables. Let us consider the case of three variables (three degrees of freedom) for simplicity in writing. The extension to the general case from this is immediate and obvious.

Then we have three parameters $u, v, w$, whose values at the start of the range have to be estimated so as to make three functions $f, g, h$ of these parameters zero at the end of the range.

Now $f=f(u, v, w), g=g(u, v, w), h=h(u, v, w)$ may be inverted to give $u=u(f, g, h), v=v(f, g, h), w=w(f, g, h)$, provided that the Jacobian of the transformation does not vanish at any of the points in question. This we may assume for any given set of numerical functions.

Denoting the values of $u, v, w, \frac{\partial u}{\partial f}, \cdots, \frac{\partial w}{\partial h}$ taken when $f=g=h=0$ by $U, V, W, U_{f}, \cdots, W_{h}$, respectively, then we obtain from Taylor's series truncated after its first-order terms

$$
u=U+f U_{f}+g U_{o}+h U_{h} .
$$

From four such equations (derived from the approximations $u_{1}, u_{2}, u_{3}, u_{4}$ )

$$
\begin{aligned}
& f_{1} U_{f}+g_{1} U_{o}+h_{1} U_{h}+U=u_{1}, \\
& f_{2} U_{f}+g_{2} U_{o}+h_{2} U_{h}+U=u_{2}, \\
& f_{3} U_{f}+g_{3} U_{o}+h_{3} U_{h}+U=u_{3}, \\
& f_{4} U_{f}+g_{4} U_{o}+h_{4} U_{h}+U=u_{4},
\end{aligned}
$$

we may eliminate $U_{f}, U_{\theta}, U_{h}$ to obtain the required interpolate $U$. This set of equations may be solved by the Gauss elimination method [3] to give $U$ directly, with no back-substitution.

Further, the sets of equations for $V$ and $W$ differ from the above set for $U$ only in that $u_{1}$ to $u_{4}$ are replaced by $v_{1}$ to $v_{4}$ and $w_{1}$ to $w_{4}$ respectively. Hence the whole process may be represented in matrix form as

$$
\left[\begin{array}{llll}
f_{1} & g_{1} & h_{1} & 1 \\
f_{2} & g_{2} & h_{2} & 1 \\
f_{3} & g_{3} & h_{3} & 1 \\
f_{4} & g_{4} & h_{4} & 1
\end{array}\right]\left[\begin{array}{lll}
U_{f} & V_{f} & W_{f} \\
U_{0} & V_{0} & W_{0} \\
U_{h} & V_{h} & W_{h} \\
U & V & W
\end{array}\right]=\left[\begin{array}{ccc}
u_{1} & v_{1} & w_{1} \\
u_{2} & v_{2} & w_{2} \\
u_{3} & v_{3} & w_{3} \\
u_{4} & v_{4} & w_{4}
\end{array}\right],
$$

and the elimination process may be carried through to give a result of the form

$$
\left[\begin{array}{lll}
F U & F V & F W
\end{array}\right]=\left[\begin{array}{lll}
\bar{u} & \bar{v} & \bar{w}
\end{array}\right],
$$

whence $U=\bar{u} / F, V=\bar{v} / F, W=\bar{w} / F$. This process may then be repeated with four sets of values comprising $(U, V, W)$ and the three best approximations from $\left(u_{1}, v_{1}, w_{1}\right), \cdots,\left(u_{4}, v_{4}, w_{4}\right)$. There is a corresponding reduction in labour if the asymmetrical Cholesky process [3] is used. 
Formal solution of the matrices gives

$$
U=\frac{\left|u_{1} f_{2} g_{3} h_{4}\right|}{\left|1 f_{2} g_{3} h_{4}\right|}
$$

which is seen to be a (formal) extension of the linear interpolation formula in the Aitken process, since the usual formula

can be rewritten

$$
f\left(u ; f_{1}, f_{2}\right)=\left(u_{1} f_{2}-u_{2} f_{1}\right) /\left(f_{2}-f_{1}\right)
$$

$$
f\left(u ; f_{1}, f_{2}\right)=\frac{\left|u_{1} f_{2}\right|}{\left|1 f_{2}\right|} .
$$

4. Example. As an example of this process, consider the steady-state solution of van der Pol's equation subject to a forcing function,

$$
\frac{d^{2} x}{d t^{2}}-\left(1-x^{2}\right) \frac{d x}{d t}+x=\cos t
$$

If $x(0)=a, x^{\prime}(0)=b$, we may be required to estimate both $a$ and $b$ so that $x(2 \pi)=a, x^{\prime}(2 \pi)=b$, that is, the solution has the same period as the forcing function. For a given $(a, b)$ let $x(2 \pi)=c, x^{\prime}(2 \pi)=d$, and define $f=a-c$, $g=b-d$. Then we may integrate forward with three independent sets of $(a, b)$

\begin{tabular}{|c|c|c|c|c|c|c|c|c|c|}
\hline & $a$ & $b$ & $c$ & $d$ & $f$ & $g$ & $a$ & $\hat{b}$ & $\begin{array}{c}\text { Computed } \\
\text { using } \\
\text { runs }\end{array}$ \\
\hline & $\begin{array}{l}+1 \\
+1 \\
+1.200 \\
+1.227 \\
+1.190\end{array}$ & $\begin{array}{l}0 \\
-1 \\
-1.600 \\
-1.411 \\
-1.448\end{array}$ & $\begin{array}{l}+1.227 \\
+1.140 \\
+1.196 \\
+1.197 \\
+1.190\end{array}$ & $\begin{array}{l}-1.411 \\
-1.499 \\
-1.443 \\
-1.441 \\
-1.448\end{array}$ & $\begin{array}{l}-0.227 \\
-0.140 \\
+0.004 \\
+0.030\end{array}$ & $\begin{array}{l}+1.411 \\
+0.499 \\
-0.157 \\
+0.030\end{array}$ & $\begin{array}{c}\overline{-} \\
+1.2271 \\
+1.1903\end{array}$ & $\begin{array}{c}\bar{Z} \\
-1.4106 \\
-1.4482\end{array}$ & $\begin{array}{l}\overline{7} \\
1,2,3 \\
2,3,4\end{array}$ \\
\hline
\end{tabular}
and then interpolate inversely to estimate values $\hat{a}, \hat{b}$ corresponding to $f=g=0$. The results are shown in Table 2 , and exhibit quite a rapid convergence.

TABLE 2

5. Conclusion. The method described above has been used on various differential equations and has shown quite good convergence. When a series of solutions with varying parameters have to be found, it is usually possible to "predict" a set of values lying near the required ones. The convergence is then more rapid.

This note is published by permission of the Director of the National Physical Laboratory.

\section{F. J. WARNER}

National Physical Laboratory

Teddington, Middlesex

England

1. L. M. Milne-Thomson, The Calculus of Finite Differences, Macmillan, London, 1933, $\S 3.81$.

2. M. C. K. TwEedIE, "A modification of the Aitken-Neville Linear iterative procedures for polynomial interpolation," $M T A C, \mathrm{v} .8,1954$, p. 13-16.

3. L. Fox, "Practical solution of linear equations and inversion of matrices," NBS Applied Mathematics Series, v. 39, 1954, p. 1-54. 